\title{
Frozen dynamics and synchronization through a secondary symmetry-breaking bifurcation
}

\author{
M. A. Miranda* \\ J. Burguete, H. Mancini, and W. González-Viñas ${ }^{\dagger}$ \\ Department of Physics and Applied Mathematics, University of Navarra, Pamplona, Spain \\ (Received 21 September 2012; revised manuscript received 22 December 2012; published 5 March 2013)
}

\begin{abstract}
We show evidence of the frozen dynamics (Kibble-Zurek mechanism) at the transition one-dimensional (1D) front of an extended 1D array of convective oscillators that undergo a secondary subcritical bifurcation. Results correspond to a global synchronization process from nonlocal coupling between the oscillating units. The quenched dynamics exhibits defect trapping at the synchronization front according to the Kibble-Zurek mechanism, predicted for condensed matter systems. A stronger subcriticality prevents the fronts from freezing defects during the quenched transitions. A synchronization model of supercritical oscillating units is proposed to explain differentiation mechanisms in morphogenesis above a critical crossing rate when the frequency of the individual oscillators becomes coherent. The phases of such oscillators are spatially coupled through a Kuramoto-Battogtokh term that leads to the experimentally observed subcriticality. As a consequence, we show that the Kibble-Zurek mechanism overcomes non-locality of a geometrical network above a critical crossing rate.

DOI: 10.1103/PhysRevE.87.032902

PACS number(s): 05.45.Xt, 64.60.aq, 05.70.Fh
\end{abstract}

\section{INTRODUCTION}

Amazing synchronization phenomena occur in nature from cellular morphogenesis in living organisms to the distribution of galaxies. We may ask how such different systems show similar dynamical responses (e.g., generation and spatiotemporal distribution of defects) when they undergo an instability towards a "new state." Precedents on synchronization are found in the pioneering work by Winfree [1] and Kuramoto [2] and in the research on collective behavior in life sciences like neurology [3], ecology [4], and networks [5-10]. However, joint experimental [11-15] and theoretical [16-18] efforts have been scarce, and more research must be devoted to the understanding of synchronization processes in complex systems from the networks approach.

Synchronization phenomena have to be limited by causal effects like the Kibble-Zurek (KZ) mechanism [19,20], which explains the appearance of topological defects in nonequilibrium symmetry-breaking second-order phase transitions. When the control parameter $\epsilon$ is varied at a given rate, $\mu$, across the critical point, the healing length $\xi$ scales as

$$
\xi\left(-t_{o}\right) \sim \mu^{-\frac{v}{1+z \nu}},
$$

where $v$ is the critical exponent of the correlation length of the order parameter $\epsilon, z$ is the dynamical critical exponent [21], and $-t_{o}$ is the time at which the dynamics gets arrested. Specially, the KZ mechanism attracts the interest of cosmologists because of its theoretical implications in an inflationary universe, regarding the distribution of frozen defects such as galaxies in an expanding universe. These cosmological defects might appear from a causal horizon which is determined by the crossing rate at the very first instants after the big bang [19,22]. There are also some theoretical approaches which deal with subcritical quenched transitions in cosmological inflationary theories [23].

\footnotetext{
*montse@alumni.unav.es

†wens@unav.es
}

The KZ mechanism has already been tested in primary bifurcations performed in 4-He systems, Josephson arrays, lasers, and thermoconvective experiments. These experiments belong to the so-called "Cosmology in the laboratory" [20, 24-26]. Concerning mathematical models, defect formation in supercritical symmetry-breaking transitions has also been reported in Refs. [27,28]. In this scenario, the present study represents a further step from the previous work in quenched convection [13,29-32].

A consistent networks description of synchronization phenomena in physics, by coupling phase and amplitude equations, would be a relevant advance in the understanding of dynamic morphologies. Such a description could be extended to other self-entrainment processes which appear in nature when assemblies of identical units interact by means of a nonlocal coupling. Besides, the consideration of the rate at which the synchronization is triggered in an extended system, which consists of a large ensemble of latent oscillators, is necessary. Consequently, a complex system may exhibit phenomena of defect trapping similar to the one predicted by Kibble-Zurek in condensed matter systems. This will occur for supercritical transitions or for very weak subcritical transitions and, in this latter case, by using quenches above a critical crossing rate. A straightforward application of these phenomena is to control the processing of nanostructured materials by choosing the proper crossing rate, aiming at controlling the self-assembly parameters such as the clustering size or the distribution of defects in patterning.

Here, we aim to prove by experiments and simulations that it is possible to recover causal frozen dynamics in a secondary symmetry-breaking subcritical bifurcation. The subcritical character of this bifurcation comes from the nonlocal interaction of the oscillating units, which is not able to counteract the $\mathrm{KZ}$ mechanism for fast crossing rates. In fact, we consider the topic of firing synchronization processes from nonlocal coupling towards new coherent patterns. In addition, the present study deals with a multicellular stationary pattern (ST) as the most symmetric phase that is able to undergo a subcritical bifurcation, which allows the coexistence of 
different patterns for a range of the control parameter. The spatiotemporal one-dimensional (1D) front which separates both phases may be considered as a synchronization front.

In our convection experiment with localized heating along a central line from below, the multicellular stationary pattern ST consists of an ensemble of stationary convective rolls as predicted by Smith and Davis in 1983 [33] with a wave vector perpendicular to the horizontal temperature gradient. As the control parameter is increased the thermal boundary layer near the heating line may destabilize in a secondary bifurcation leading to an oscillatory pattern [34]. Each roll corresponds to a hot spot in the bulk of a thin fluid layer along the quasi-1D heating line (for a detailed explanation see Refs. [12,35-37]). It is possible to reduce the degrees of freedom of the system to those of the hot spots [12], hereafter named convective oscillators because the secondary symmetry-breaking bifurcation supplies each hot spot with a natural frequency, $\omega_{n}$, in the new oscillatory pattern. As a consequence of the degrees of freedom reduction, a nonlocal interaction between the convective oscillators emerges, which is in charge of the great diversity of synchronization phenomena, including "chimera states" [17] when the convective oscillators become coherent in coexistence with the ST pattern beyond a spatiotemporal chaotic regime. In these chimera states, the nonlocal interaction range increases inside the coherent oscillatory patterns because the amplitude of the critical modes becomes stronger in the new coherent state [36,37]. From these results, we stressed the importance of describing a spatiotemporal synchronization transition from the coupling between the amplitude of the critical modes and their phases. In the framework of the $\mathrm{KZ}$ mechanism, the correlation length is given by the average length between frozen defects. These frozen defects are trapped topological singularities which, in our experiment, correspond to phase defects that appear along the synchronization front that separates the ST pattern from the new one.

In this article, a networks model of spatio-temporal synchronization through a quenched secondary bifurcation is presented together with experimental results. We show that frequency locking above a critical quenched transition in the end renders the KZ mechanism. Finally, it is stressed that the classical Kuramoto model of global synchronization does not take into account the differentiation processes that arise from the rate at which the instability is crossed, though mechanisms like the $\mathrm{KZ}$ one are to be encountered in weakly subcritical bifurcations. Furthermore, the transition 1D fronts spread along the space-time giving rise to a new "phase" which keeps registering frozen defects depending on the relaxation dynamics that comes from the interaction between convective oscillators. In this new phase, the mean-field approach of Kuramoto [2] can be also checked.

\section{QUENCHED DYNAMICS IN THE THERMOCONVECTIVE EXPERIMENT}

Our experiment is a large 1D array of around 100 convective oscillators which are hydrodynamically coupled. These convective oscillators, in the latent state (prior to the quench) configure the stationary multicellular pattern ST. The convective oscillators' dynamics is recorded over the 50 central ones by using a shadowgraphy technique. A sketch of a

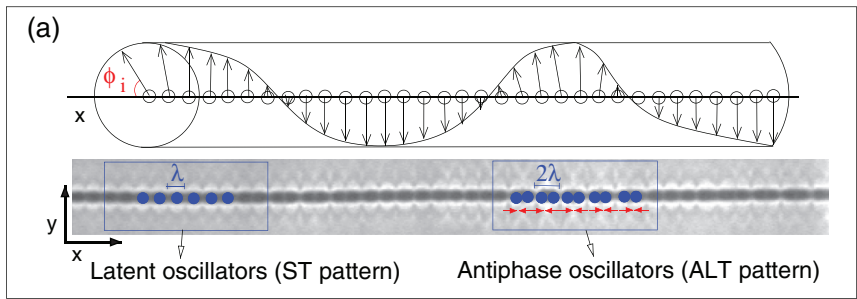

(b)

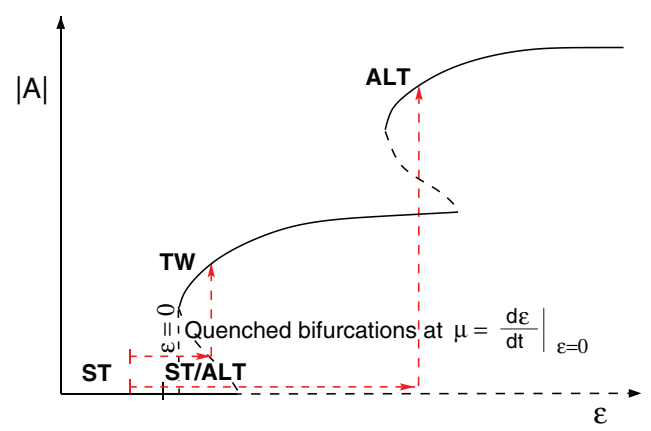

(c)

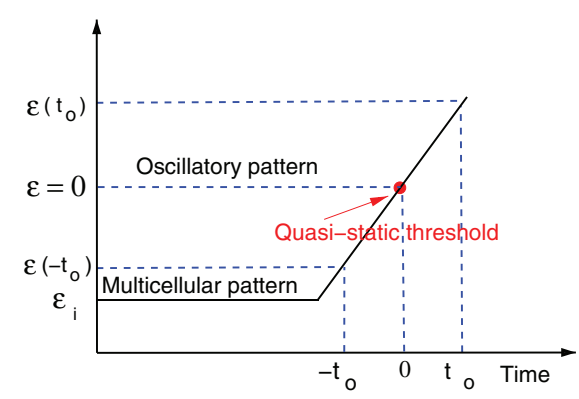

FIG. 1. (Color online) (a) Sketch of the 1D array of coupled oscillators (each oscillator is given by a convective cell) whose coherent phases describe a long wavelength profile. Bottom: A shadowgraphy image of the convective cell corresponding to the oscillatory cells in the antiphase (ALT) pattern coexisting with latent oscillatory cells (ST pattern). The average wavelength of the stationary pattern is approximately $4.6 \mathrm{~mm}$ and becomes doubled in the ALT. (b) Sketch of the two subsequent secondary subcritical bifurcations from the ST pattern towards the ALT pattern. Dashed arrows depend on the crossing rate value. (c) Quasistatic threshold $(\epsilon=0)$ at a secondary symmetry-breaking instability from a multicellular pattern $\left(\epsilon_{i}\right.$ is the initial control parameter value) towards an oscillatory pattern.

coherent behavior of the phases of a 1D array of convective oscillators, which are characterized as limit-cycle oscillators, is shown in Fig. 1(a). Below this sketch, a shadowgraphy image from the experiment where antiphase oscillators (alternating pattern, ALT) coexist with latent oscillators (ST pattern) is also shown. By modifying the control parameter $(\epsilon)$, which is the reduced vertical temperature difference for a fixed depth of the fluid layer, the ST pattern is going to be driven throughout one or two subsequent secondary bifurcations depending on the quench rate. According to the bifurcation diagram sketched in Fig. 1(b), if the system quasiadiabatically crosses the threshold $(\epsilon=0)$ the latent oscillators (ST pattern) become active oscillators which may perform antiphase (ALT pattern) and in-phase (traveling waves pattern, TW) synchronization. Coexistence between these patterns in the case of ST/ALT + TW and TW + ALT below a Co- 2 point and a detailed explanation of the stability diagram structure 


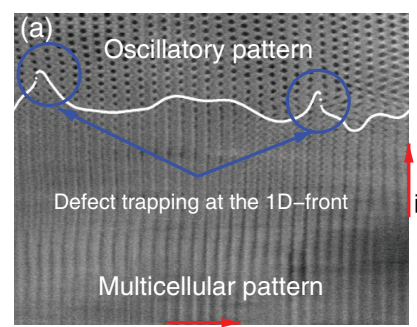

Space

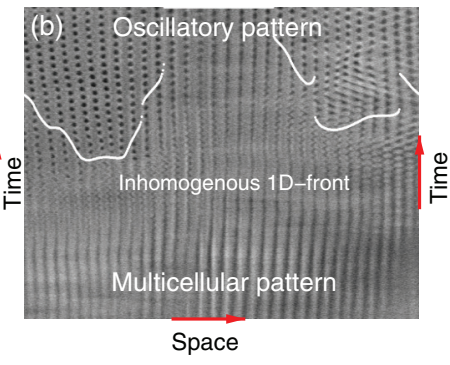

FIG. 2. (Color online) Experimental spatiotemporal diagrams (the average recording time is $15 \mathrm{~min}$ along with roughly 50 convective cells) of quenched transitions for (a) a weak subcritical secondary bifurcation (at $d=7.5 \mathrm{~mm}$, from $36{ }^{\circ} \mathrm{C}$ towards $52{ }^{\circ} \mathrm{C}$ ), where two topological defects are trapped at the transition 1D front, and (b) a strong subcritical secondary bifurcation (at $d=7 \mathrm{~mm}$, from $38^{\circ} \mathrm{C}$ towards $50^{\circ} \mathrm{C}$ ) with a nonhomogeneous $1 \mathrm{D}$ front. The transition $1 \mathrm{D}$ fronts are defined at $e^{-1}$ of the maximum critical amplitude.

have been previously reported (see Ref. [13], and references therein). In accordance with that work, it should be noticed from Fig. 1(b) that the ST/ALT pattern, which already appears underneath the threshold of the TW pattern, is a mixed pattern because it takes the form of irregular patches in the ALT pattern spread over the ST pattern. As the speed at which the threshold $[\epsilon=0$ in Fig. 1(b)] is crossed increases, the system bifurcates towards the ALT pattern as a secondary doubling wavelength bifurcation with the presence of a homogeneous synchronization 1D front except for the trapped defects [13]. In Fig. 1(c) we show the quasistatic threshold as a function of time, when the slowed down relaxation dynamics takes place $\left[-t_{o}, t_{o}\right]$, at a given crossing rate $\left(\left.\mu \equiv \frac{d \epsilon}{d t}\right|_{\epsilon=0}\right)$. Trapped defects at the synchronization front are the result of causality when, at a critical crossing rate, $\mu_{c}$, the fluctuations of the system have not had enough time to disappear and hence they become frozen at the 1D front. In Fig. 2(a) a typical spatiotemporal diagram from the experiment is shown, where two defects become trapped at the transition 1D front. Previous experiments on the $\mathrm{KZ}$ mechanism for a weak subcritical regime, which correspond to a depth of the fluid layer of $d=$ $7.5 \mathrm{~mm}$, are reported in Ref. [13]. The quenched dynamics is inferred from the homogeneity of the transition 1D front, and thus, the correlation length of the 1D front accounts for the phenomenon of defect appearance at different crossing rates. A detailed explanation of the 1D front signal analysis of the experiments can be found in Ref. [13].

Results on the quenched dynamics, regarding the correlation length $\xi$ of the 1D front versus the crossing rate $\mu$, are shown in Fig. 3(a) for two different depths of the fluid layer. Depending on the distance to the nearby Co-2 point from below, these depths correspond to two different strengths of the subcritical bifurcation: weak for $d=7.5 \mathrm{~mm}$ and strong for $d=7 \mathrm{~mm}$. In the former case, for crossing rates higher than a critical one, $\mu_{c}$ (fast quenches), defects are trapped at the 1D fronts [Fig. 2(a)]. In consequence, results for $\mu \geqslant \mu_{c}$ in Fig. 3(a) can be fitted to a typical KZ power law for the correlation length $\xi \sim \mu^{-2.8 \pm 0.1}$ (the same value is reported but for fewer sequences in Ref. [13]). In the latter case, the system exhibits always inhomogeneous $1 \mathrm{D}$ fronts regardless of the crossing rate [Fig. 2(b)]. Due to this intrinsic heterogeneity,
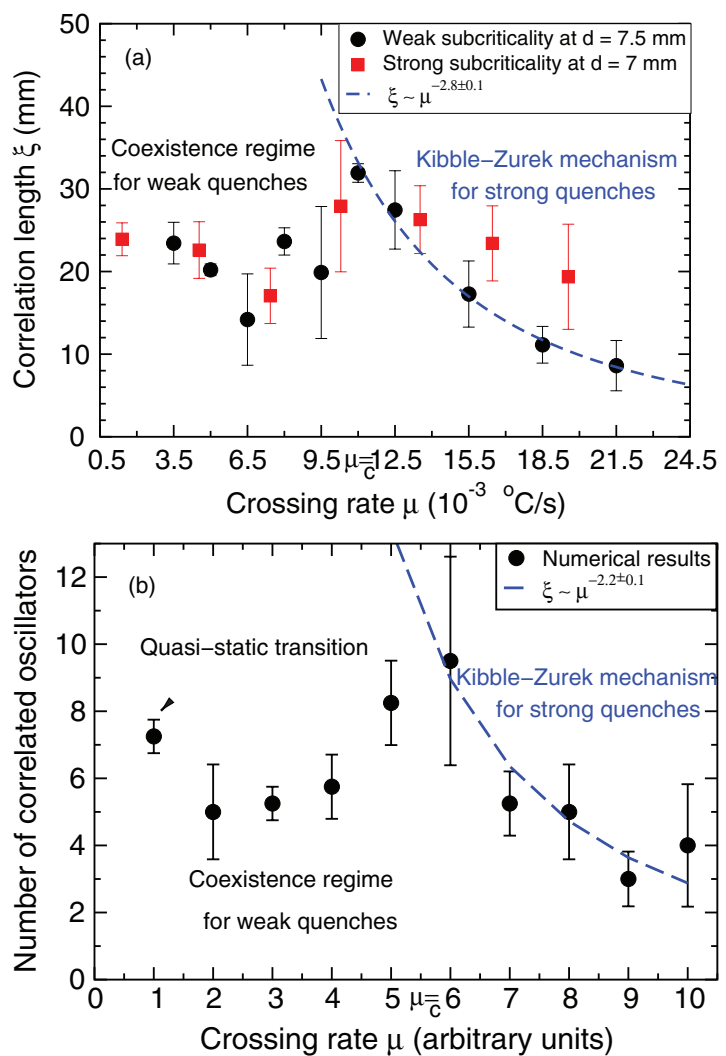

FIG. 3. (Color online) (a) Experimental results on the correlation length vs the crossing rate for a secondary weak subcritical bifurcation ( $d=7.5 \mathrm{~mm}$, circles) and for a strong secondary subcritical bifurcation ( $d=7 \mathrm{~mm}$, squares). The dashed line for $\mu \geqslant \mu_{c}$ (where $\mu_{c}$ is the critical crossing rate for the most homogeneous front) is fitted to the power law $\xi \sim \mu^{-2.8 \pm 0.1}$ for the secondary weak subcritical bifurcation. (b) Numerical results on the correlation length vs the crossing rate. The dashed line for $\mu \geqslant \mu_{c}$ (where $\mu_{c}$ is the critical crossing rate for the most homogeneous front) is fitted to the power law $\xi \sim \mu^{-2.2 \pm 0.1}$.

which comes from the nonlocality in the interaction among convective oscillators, the system does not exhibit the KZ behavior [Fig. 3(a)].

\section{THE MODEL: SPATIOTEMPORAL SYNCHRONIZATION OF A 1D ARRAY OF NONLOCALLY COUPLED OSCILLATORS}

Secondary symmetry-breaking instabilities where the wavelength of the new pattern becomes doubled in a continuum field have been theoretically tackled from the normal form of the amplitude and phase equations [38,39]; however they involve too many complex parameters. This drawback can be overcome if the problem is approached from the networks framework.

To model our experimental system, we consider an ensemble of convective oscillators according to our previous work $[12,37]$. There, we showed that at the onset of a secondary bifurcation the convective oscillators were nonlocally coupled (with an interaction range of about five or six oscillators). In addition, the background on quenched transitions that exhibit the KZ mechanism at the synchronization front [13] allows us 
to model this spatiotemporal synchronization transition from the coupling between the amplitude of the critical modes, which shape the synchronization front, and their phases.

Our spatiotemporal synchronization model is inspired by the Kuramoto-Battogtokh model [40] which takes into account a large assembly of limit-cycle oscillators with nonlocal coupling. The nonlocal coupling is defined by the coupling constant $K$ and the Green's function $G\left(x-x^{\prime}\right)$. In the continuum limit a general phase model equation is given by

$$
\frac{\partial}{\partial t} \phi(x, t)=\omega-K \int d x^{\prime} G\left(x-x^{\prime}\right) \sin \left[\phi(x, t)-\phi\left(x^{\prime}, t\right)+\alpha\right] \text {. }
$$

In our model, the discrete Green's function is chosen as a Lorentzian because it accomplishes the condition of a linear continuous functional. These Lorenztian functions, $K_{i j}$, also provide an interaction strength, of the repulsive type, between the convective oscillators that can be determined experimentally [12]. The repulsive interaction between oscillators corresponds to the characteristic antiphase oscillation (alternating pattern). To quantify the degree of interaction between each $i j$ pair of oscillators, we introduce a coupling magnitude, $\sigma$, into the final interaction strength (where $\gamma$ accounts for the diffusion range):

$$
K_{i j}=\frac{-\sigma \gamma}{\pi\left(\gamma^{2}+\left(\left|x_{j}-x_{i}\right|\right)^{2}\right)} .
$$

The subcritical nature of our system, close to the Co-2 point, is approached by the complex quintic Ginzburg-Landau equation (cQGLE) [41]. This equation accomplishes the symmetries of the system and succeeds in reproducing the dynamics of coherent and localized structures [42,43]. Although the need to include nonlocal terms in the cQGLE has been previously considered [44], the diffusion term in the discrete space allows us to introduce the nature of the nonlocal coupling in our model, as is pointed out in the following. Moreover, the quintic term is able to stabilize localized solutions in a subcritical framework. Under these circumstances, to face the quenched dynamics, these amplitude equations (cQGLE) is introduced as a step further than the previous spatiotemporal phase synchronization transition $[12,36]$. Close to the onset of the secondary subcritical bifurcation, the phase is nonlinearly coupled with the new unstable amplitude. Therefore, we consider that each convective oscillator $(i)$ can be described via its phase $\phi_{i}$ and its amplitude $\rho_{i}$. Then, the model consists of an ensemble of cQGLEs:

$$
\begin{aligned}
\tau\left(\partial_{t} A_{j}\right)= & \left(\epsilon_{o}+i \omega_{n}\right) A_{j}+\left(b_{3}+i c_{3}\right)\left|A_{j}\right|^{2} A_{j}- \\
& -\left(b_{5}-i c_{5}\right)\left|A_{j}\right|^{4} A_{j}+\left(b_{1}+i c_{1}\right) \partial_{x x} A_{j}
\end{aligned}
$$

Each equation provides the usual amplitude-phase description $A_{j}=\rho_{j} e^{i \phi_{j}}$. The control parameter is $\epsilon_{o}=\frac{T_{c}-T}{T_{c}}$ (where $T_{c}$ is the critical temperature at the onset of a secondary subcritical instability), and the natural frequency associated with each $j$ oscillator is $\omega_{n}$. However, from this equation the straightforward way to approach the 1D front dynamics is to impose supercritical conditions on the individual oscillators ( $b_{3}<0$ and $b_{5}>0$ [41]) in order to achieve the homogeneous $1 \mathrm{D}$ front at the critical crossing rate. Therefore, once the critical crossing rate is exceeded, the system exhibits defect trapping at the 1D front as is expected [31]. This is consistent with the fact that the oscillators synchronize with the restriction from the causal effect (arrested dynamics) led by the $\mathrm{KZ}$ mechanism. Nevertheless, it is below the critical crossing rate that the weak subcritical nature of the system, which involves coexistence with a fluctuating transition 1D front, emerges from the nonlocal coupling between oscillators. At the end of this section this issue is taken up again.

The diffusion is controlled by the last term of Eq. (1), where we generalize $\partial_{x x} A_{j}$ to the discrete form $\sum_{j \neq i} K_{j i}\left(A_{j}-A_{i}\right)$. Not only is this the standard coupling for systems with several subsystems near synchronization [45], but it is also a suitable discrete form of $\partial_{x x} A_{j}$ by choosing the appropriate $K_{j i}$. The numerical work consists of implementing the second-order Adams-Bashforth method for the phase and the amplitude equations.

Finally, the model equations for an ensemble of $i$ coupled oscillators with dragging frequency $\tilde{\omega}_{o i}$ and control parameter $\tilde{\epsilon}_{o i}$ become

$$
\begin{aligned}
\partial_{t} \phi_{i}= & \tilde{\omega}_{o i}+c_{3} \rho_{i}^{2}+c_{5} \rho_{i}^{4} \\
& +\chi \sum_{j \neq i} K_{j i} \rho_{j} \sin \left(\phi_{j}-\phi_{i}+\beta\right) \frac{1}{\rho_{i}}, \\
\partial_{t} \rho_{i}= & \tilde{\epsilon}_{o i} \rho_{i}+b_{3} \rho_{i}^{3}-b_{5} \rho_{i}^{5} \\
& +\chi \sum_{j \neq i} K_{j i} \rho_{j} \cos \left(\phi_{j}-\phi_{i}+\beta\right),
\end{aligned}
$$

with

$$
\beta=\arctan \frac{c_{1}}{b_{1}} . \quad \chi=\sqrt{b_{1}^{2}+c_{1}^{2}},
$$

where the control parameter $\tilde{\epsilon}_{o i}$ and the dragging frequency $\tilde{\omega}_{o i}$ are related to the nonlocal coupling $K_{i j}$ by

$$
\tilde{\epsilon}_{o i}=\epsilon_{o}-b_{1} \sum_{j \neq i} K_{j i}, \quad \tilde{\omega}_{o i}=\omega_{n}-c_{1} \sum_{j \neq i} K_{j i} .
$$

This model, with periodic boundary conditions and random initial conditions, allows us to reproduce quasiadiabatic bistable patterns of the system above two subsequent subcritical instabilities $[35,36]$ corresponding to the mixed pattern ST/ALT $\left(0<b_{5}<1\right)$, where isolated clusters of synchronized oscillators are spread over the stationary multicellular pattern $\mathrm{ST}$, and the TW + ALT patterns $\left(b_{5}=1\right)$, where both in-phase and antiphase patterns coexist [12]. Also, the degree of coexistence between the oscillating phase and the stationary one can be modified by varying the parameter $c_{3}$ for negative values, and thus, as $\left|c_{3}\right|$ diminishes, the amplitude of the oscillators is prevented from damping and a more stable pattern is obtained. With regard to the quenched transitions, the parameter $c_{3}$ is especially relevant, because it allows us to lock the frequency of oscillation when the antiphase regime is achieved as it happens in the experiments [35] and also in the quenched dynamics above a critical crossing rate [13]. If we look for the condition of existence of the amplitude solution from the phase equation [Eq. (2)] for very small phase shifts, we get a quadratic dependence of the frequency on the coefficient $c_{3}$ from the inequality $\tilde{\omega}_{o i} \gtrsim c_{3}^{2} / 4 c_{5}$. This fact allows us to implement a feedback loop to lock the frequency of the oscillators by fixing $c_{3}$. In addition, the phase mismatch $\beta$ determines the degree of coexistence between the TW and the ALT patterns, meanwhile the modulus of the diffusion 
coefficient $\chi$ takes part in the amplitude-phase coupling by modifying the nonlocal interaction. As a consequence, proper parameters $(\chi, \beta)$ have to be chosen to numerically describe the experimental system with a null group velocity at the onset of this absolute instability [35,37].

In addition, if we consider the alternating solution $A_{i \pm 1}=-A_{i}$ in the amplitude equation [Eq. (3)], the control parameter in the approximation to nearest neighbors is given by

$$
\epsilon_{o} \approx-\frac{b_{3}^{2}}{4 b_{5}}-\frac{\sigma \gamma}{\pi\left(\gamma^{2}+1\right)}\left(b_{1}+2 \chi\right) .
$$

Therefore, it can be noticed from this expression that the nonlocal coupling has a key role in the dynamics of these kind of systems, and it must be taken into account in synchronization transitions between weakly coupled oscillating units. In this study, we have also checked out that, as is expected [46], white noise enhances synchronization in our simulations.

Under these conditions, the parameters which better describe adiabatically our weak secondary subcritical transition are $b_{1}=1, c_{1}=0.087, b_{3}=-0.1, c_{3}=-2, b_{5}=1, c_{5}=$ 0.1 , and $\omega_{n}=2 \pi 1.25$, with $\sigma=1.4$ and $\gamma=0.4$ for minor subassemblies of approximately 6 coupled oscillators. For these parameter values, and by considering a network of 50 oscillators, the secondary bifurcation threshold, from the stationary pattern ST towards the new oscillating pattern, is obtained at $\tilde{\epsilon}_{o, c}=-0.78$. This is the quasistatic threshold for a null crossing rate at the secondary weak subcritical bifurcation.

In our system, it is possible to define an effective control parameter, which locally takes into account the overall coefficient for the linear terms for $\rho_{i}$ in the right-hand side of the Eq. (3). As a consequence, for slow quenches (below the critical crossing rate) the simulations show coexistence that is triggered by local excursions of the effective control parameter towards positive values. Moreover, the nonlocal interaction between supercritical oscillators, which has been introduced by means of the interaction strength $K_{i j}$, accounts for the observed subcritical dynamics.

\section{QUENCHED DYNAMICS IN THE SYNCHRONIZATION MODEL: DISCUSSION OF RESULTS}

Here we focus on the quenched dynamics which is performed by launching the numerical simulation from below the critical value $\left(\tilde{\epsilon}_{o, c}=-0.78\right)$ at the initial control parameter $\left(\tilde{\epsilon}_{o, \min }=-1.7\right)$ until the system is settled slightly above it at the final control parameter $\left(\tilde{\epsilon}_{o, \max }=-0.6\right)$. These quenched transitions are performed with different values of the crossing rate $\mu$ ranging from $\mu_{\min }=0.05$ to $\mu_{\max }=10$ (in arbitrary units). The range of $\mu$ is determined to ensure that for the fastest quench a minimum of 100 steps are to be performed; therefore we choose a compromise between time discretization $(\Delta t=0.001)$ and an acceptable quench rate range, that is, $\mu_{\max }=\left(\tilde{\epsilon}_{o, \max }-\tilde{\epsilon}_{o, \min }\right) /(100 \Delta t)$. The quenched dynamics is triggered after an initial transient to allow the system to settle first into the ST state.

As the coefficient $c_{3}$ boosts the dragging force to install synchronization in the antiphase pattern beyond the synchronization front, we choose the ansatz of a linear dependence of a new parameter, $c_{3}^{\prime}$, on the quench rate in the form $c_{3}^{\prime}=$ $\left.c_{3}\left[1-\left(\mu / \mu_{\min }\right) \Delta \mu\right)\right]$ (with step $\left.\Delta \mu=0.005\right)$ until the most
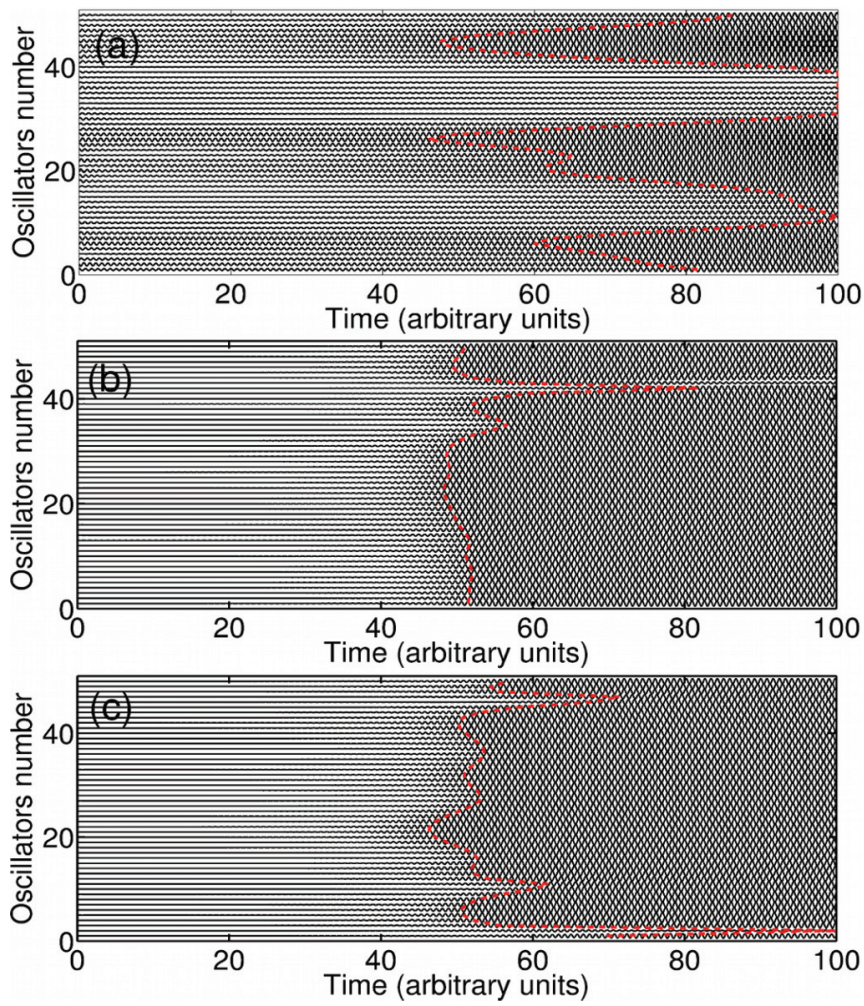

FIG. 4. (Color online) Spatiotemporal diagrams obtained from the numerical simulation at different crossing rates (arbitrary units): (a) $\mu=0$ (quasiadiabatic transition), (b) $\mu=\mu_{c}=6$, (c) $\mu=10$.

homogeneous 1D front is formed at $\mu=\mu_{c}=6$ [Figs. 4(b) and 5(a)]. The physical interpretation of the ansatz is that the linear dependence of $c_{3}^{\prime}$ on the quench rate guarantees the synchronization frequency to spread along the 1D array within the range $\mu=\left[0, \mu_{c}\right]$. Therefore, when the homogeneous $1 \mathrm{D}$ front is able to freeze defects, $c_{3}^{\prime}$ is kept constant at -0.8 . According to this, the numerical results provide a frequency distribution along the $1 \mathrm{D}$ array for $\mu \geqslant \mu_{c}$ of $\approx(0.35 \pm 0.24) \mathrm{s}^{-1}$, while below $\mu_{c}$ the frequency of synchronization is higher with a larger dispersion $\left[\approx(0.45 \pm 0.35) \mathrm{s}^{-1}\right]$; these results agree with the experimental behavior of frequencies [13]. A zoomed spatiotemporal diagram from the numerical simulation at $\mu_{c}$ is shown in Fig. 6. In this figure, the 1D front that bounds the new
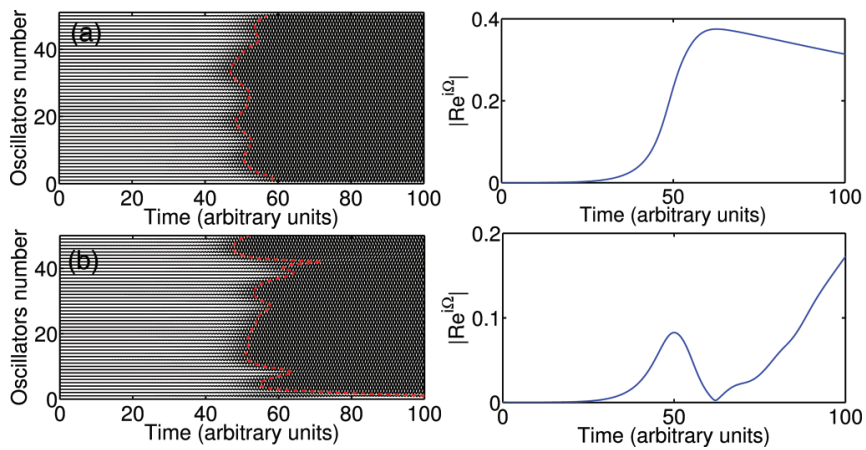

FIG. 5. (Color online) On the left spatiotemporal diagrams from the numerical simulations with the corresponding $1 \mathrm{D}$ front, and on the right a mean-field approach of the synchronization process beyond the threshold in panel (a) at $\mu=6$ and in panel (b) at $\mu=8$. 


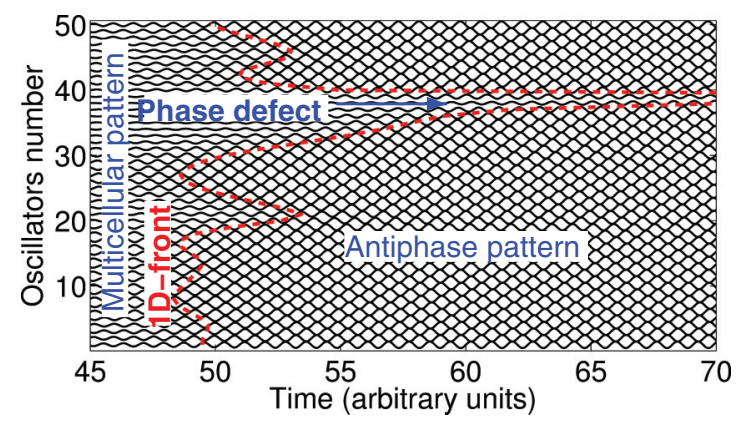

FIG. 6. (Color online) Spatiotemporal diagram obtained from the numerical simulation at $\mu_{c}=6$. The $1 \mathrm{D}$ front, which is defined at $e^{-1}$ of the maximum amplitude, splits the diagram into the multicellular pattern and the new antiphase pattern.

antiphase pattern exhibits a frozen defect on it. Consequently, the larger error bar obtained at $\mu=\mu_{c}=6$ [see Fig. 3(b)] is due to the fact that the system may already exhibit defect trapping at this stage [see Figs. 6 and 4(b)].

From the numerical simulations, the dynamics of the convective oscillators at different crossing rates is shown in Fig. 4 under the form of spatiotemporal diagrams which resemble the quenched experimental results [13]. Also, in this figure, the evolution of the transition 1D front with the increasing value of the crossing rate can be observed, as well as the trapped defects. From these diagrams, the maximum value of the standard deviation of the 1D front is obtained at $\mu=0$ by launching the simulation slightly above the critical threshold $\tilde{\epsilon}_{o, c}$ [Fig. 4(a)], which corresponds to a quasiadiabatic transition. Once the homogeneous 1D front is achieved at $\mu=\mu_{c}$, the $\mathrm{KZ}$ mechanism is recovered. For $\mu \geqslant \mu_{c}$, although the frozen defects disturb the dynamics of the $1 \mathrm{D}$ front, the loss of spatiotemporal coherence cannot be measured from the standard deviation of the 1D front [13]. Therefore, the correlation length at the $1 \mathrm{D}$ fronts is determined from their self-correlation. However, to disregard the contribution of the modulations, which are inherited from the numerical method, for crossing rates higher than $\mu_{c}$ the correlation function is evaluated at the second correlation peak.

In Fig. 3(b) the self-correlation of the 1D front provides the degree of synchronization between the oscillators. These numerical results are similar to the ones obtained in experiments for weak subcriticality conditions [Fig. 3(a)]. In Fig. 3(b), for slow quenches the system shows a smooth increase of the correlation from the quasiadiabatic transition [see an example in Fig. 4(a)] until $\mu_{c}$ is reached. Above $\mu_{c}$, the quenched dynamics exhibits the "freezing effect" of the $\mathrm{KZ}$ mechanism. Accordingly, as the crossing rate increases the number of trapped defects (phase singularities) at the transition 1D front increases up to three or four defects [see the numerical spatiotemporal diagram for the maximum crossing rate in Fig. 4(c)]. For $\mu \geqslant \mu_{c}$, according to the KZ mechanism a correlation length with a critical exponent of $v=2.2$ is obtained: $\xi \sim \mu^{-2.2 \pm 0.1}$ [see Fig. 3(b)]. From these results it is inferred that the $\mathrm{KZ}$ mechanism, for weak subcriticality, is able to overcome the effects of nonlocality above a critical crossing rate.

In addition, it is possible to measure the evolution of these singularities in the aged system far from the threshold, where the dynamics must depend on the characteristic diffusion spatiotemporal scales given by the nonlocal coupling. Therefore, the synchronization processes behind the $1 \mathrm{D}$ front can be analyzed by measuring the degree of synchronization given by the mean-field approach of Kuramoto [2]:

$$
R(t) e^{i \Omega(t)} \equiv \frac{1}{N} \sum_{j} \rho_{j} e^{2 i \phi_{j}},
$$

where the factor 2 in the phase is introduced to follow the interaction range between pairs of coupled units that exhibit the antiphase mode. Hence, the modulus value of this expression allows us to measure the "dragging trend" between oscillators far from the threshold. An example is shown at the critical crossing rate $\mu=6$ in Fig. 5(a) and for a fast quench $\mu=8$ in Fig. 5(b). From these results, we can infer that the dragging trend is a measure of the degree of antiphase coherence in the final state which is fostered under fast quenches.

\section{CONCLUDING REMARKS}

The quenched dynamics through a secondary symmetrybreaking bifurcation of a 1D-array of nonlocally coupled convective oscillators yields a transition 1D front that splits two different regimes: the stationary cellular pattern (ST) from the new oscillatory pattern, which is characterized by the coexistence between in-phase (TW) and antiphase (ALT) regimes. The degree of spatiotemporal correlation of these synchronization fronts is inherited from the crossing rate $\mu$ at the core of this secondary bifurcation and agrees with the $\mathrm{KZ}$ mechanism for fast quenches $\left(\mu \geqslant \mu_{c}\right)$ in the weak subcritical regime (close to the Co-2 point). The frozen defects caused by the $\mathrm{KZ}$ mechanism appear in the form of phase singularities which become trapped at the transition 1D front for fast quenches.

According to the experimental results, a new model of spatiotemporal synchronization consisting of coupled phaseamplitude equations associated with each oscillator enables the 1D front to trap defects for fast quenches $\left(\mu \geqslant \mu_{c}\right)$. Because at $\mu \geqslant \mu_{c}$ the frequency of the oscillators becomes locked in the antiphase regime, the most correlated $1 \mathrm{D}$ front is obtained at $\mu_{c}$. Therefore, it is justified to make the ansatz of fixing the frequency-related parameter $\left(c_{3}\right)$ in the model. Under this assumption, the numerical results agree with the experiments.

It is worth noting that the nonlocal interactions among the individually supercritical oscillators give raise to a subcritical bifurcation. In our approach, the coexistence of states is influenced by the coupling between amplitude and phase equations, which comes naturally from the complex model equation [Eq. (1)] instead of considering an ad hoc Kuramoto equation [47]. According to this, it has to be mentioned that we experimentally obtain the time scale $\left(\sim 1 / \mu_{c}\right)$ at which the causal decorrelation given by the $\mathrm{KZ}$ mechanism overcomes the spatial range of nonlocality in our geometrical network.

Furthermore, we suggest that the $\mathrm{KZ}$ mechanism might be viewed as a differentiation mechanism in pattern formation or morphogenesis, because it promotes differentiation behind a synchronization front depending on the transition crossing rate. Collective synchronization in populations of nonlocally 
coupled units (e.g., individuals of an ecosystem, physical and chemical oscillators, ...) may exhibit this differentiation mechanism when a bifurcation towards a new coherent state is quenched above a critical crossing rate. Finally, our results could be generalized to understand the appearance of chimera states in a purely homogeneous network $[14,15]$ if the latent oscillators of our system are to be understood as chaotic oscillators with yet undetermined phases before the synchronization is fired over bounded regions of spatiotemporal coherence.

From a practical point of view, this study can be applied to control self-organization processes through secondary bifurcations to target new patterns avoiding the presence of defects, even in quenched regimes, for crossing rates below the critical value $\mu_{c}$. On the other hand, the cosmological implications of this theory and experiment, under quenched transitions, allow us to support causal arguments, like the $\mathrm{KZ}$ one, as plausible mechanisms for the distribution of defects across the universe even through a secondary and weakly subcritical bifurcation.

\section{ACKNOWLEDGMENTS}

This work has been partly supported by the Spanish Contracts No. FIS2008-01126 and No. FIS2011-24642 and by the Gobierno of Navarra (Departamento de Educación).
[1] A. T. Winfree, J. Theor. Biol. 16, 15 (1967).

[2] Y. Kuramoto, Chemical Oscillations, Waves and Turbulence (Dover, New York, 2003).

[3] Q. Y. Wang, Z. S. Duan, L. Huang, G. R. Chen, and Q. S. Lu, New J. Phys. 9, 383 (2007).

[4] M. Ballerini, N. Cabibbo, R. Candelier, A. Cavagna, E. Cisbani, I. Giardina, V. Lecomte, A. Orlandi, G. Parisi, A. Procaccini, M. Viale, and V. Zdravkovic, Proc. Natl. Acad. Sci. USA 105, 1232 (2008).

[5] S. Boccaletti, Y. Moreno, M. Chavez, and D.-U. Hwang, Phys. Rep. 424, 175 (2006).

[6] J. Gómez-Gardeñes, Y. Moreno, and A. Arenas, Phys. Rev. Lett. 98, 034101 (2007).

[7] G. V. Osipov, J. Kurths, and C. Zhou, Synchronization in Oscillatory Networks (Springer-Verlag, Berlin, 2007).

[8] A. Arenas, A. Díaz-Guilera, J. Kurths, Y. Moreno, and C. Zhou, Phys. Rep. 469, 93 (2008).

[9] H. Mancini and G. Vidal, Eur. Phys. J. D 62, 57 (2011).

[10] C. Zhou and J. Kurths, Phys. Rev. Lett. 88, 230602 (2002).

[11] D. Mertens and R. Weaver, Phys. Rev. E 83, 046221 (2011).

[12] M. A. Miranda and J. Burguete, Int. J. Bifurcation Chaos Appl. Sci. Eng. 20, 835 (2010).

[13] M. A. Miranda, J. Burguete, W. González-Viñas, and H. Mancini, Int. J. Bifurcation Chaos Appl. Sci. Eng. 22, 1250165 (2012).

[14] M. Tinsley, S. Nkomo, and K. Showalter, Nat. Phys. 8, 662 (2012).

[15] A. Hagerstrom, T. Murphy, R. Roy, P. Hövel, I. Omelchenko, and E. Schöll, Nat. Phys. 8, 658 (2012).

[16] S. I. Shima and Y. Kuramoto, Phys. Rev. E 69, 036213 (2004).

[17] D. M. Abrams and S. H. Strogatz, Phys. Rev. Lett. 93, 174102 (2004).

[18] D. M. Abrams and S. H. Strogatz, Int. J. Bifurcation Chaos Appl. Sci. Eng. 16, 21 (2006).

[19] W. H. Zurek, Nature (London) 317, 505 (1985).

[20] W. H. Zurek, Phys. Rep. 276, 177 (1996).

[21] D. I. Uzunov, Introduction to the Theory of Critical Phenomena (World Scientific, Singapore, 1993).

[22] T. W. B. Kibble, J. Phys. A 9, 1387 (1976).
[23] T. Vachaspati, Kinks and Domain Walls (Cambridge University Press, Cambridge, UK, 2006).

[24] T. W. B. Kibble, Phys. Today 60, 47 (2007).

[25] S. Ducci, P. L. Ramazza, W. González-Viñas, and F. T. Arecchi, Phys. Rev. Lett. 83, 5210 (1999).

[26] I. Chuang, R. Dürrer, N. Turok, and B. Yurke, Science 251, 1336 (1991).

[27] J. Dziarmaga, P. Laguna, and W. H. Zurek, Phys. Rev. Lett 82, 4749 (1999).

[28] P. Laguna and W. H. Zurek, Phys. Rev. Lett. 78, 2519 (1997).

[29] S. Casado, W. González-Viñas, H. Mancini, and S. Boccaletti, Phys. Rev. E 63, 057301 (2001).

[30] S. Casado, W. González-Viñas, and H. Mancini, Phys. Rev. E 74, 047101 (2006).

[31] S. Casado, W. González-Viñas, S. Boccaletti, P. L. Ramazza, and H. Mancini, Eur. Phys. J.: Spec. Top. 146, 87 (2007).

[32] W. González-Viñas, S. Casado, J. Burguete, H. Mancini, and S. Boccaletti, Int. J. Bifurcation Chaos Appl. Sci. Eng. 11, 2887 (2001).

[33] M. K. Smith and S. H. Davis, J. Fluid Mech. 132, 119 (1983).

[34] J. Burguete, D. Maza, and H. L. Mancini, Phys. D 174, 56 (2003).

[35] M. A. Miranda and J. Burguete, Phys. Rev. E 78, 046305 (2008).

[36] M. A. Miranda and J. Burguete, Phys. Rev. E 79, 046201 (2009).

[37] M. A. Miranda, Ph.D. thesis, University of Navarra, 2009.

[38] L. Gil, Europhys. Lett. 48, 156 (1999).

[39] L. Gil, A. Petrossian, and S. Residori, Phys. D 166, 1 (2002).

[40] Y. Kuramoto and D. Battogtokh, Nonlinear Phenom. Complex Syst. 5, 380 (2002).

[41] M. Cross and P. Hohenberg, Rev. Mod. Phys. 65, 851 (1993).

[42] R. J. Deissler and H. R. Brand, Phys. Rev. Lett. 72, 478 (1994).

[43] P. Gutiérrez, D. Escaff, S. Pérez-Oyarzún, and O. Descalzi, Phys. Rev. E 80, 037202 (2009).

[44] I. Melbourne, J. Nonlinear Sci. 8, 1 (1998).

[45] L. M. Pecora and T. L. Carroll, Phys. Rev. Lett. 64, 821 (1990).

[46] B. Lindner, J. García-Ojalvo, A. Neiman, and L. SchimanskyGeier, Phys. Rep. 392, 321 (2003).

[47] J. Gómez-Gardeñes, S. Gómez, A. Arenas, and Y. Moreno, Phys Rev. Lett. 106, 128701 (2011). 